\title{
The respiratory oscillation in yeast phase definitions and periodicity
}

\section{Douglas B. Murray}

In response to the article by $\mathrm{Tu}$ and McKnight ${ }^{1}$, I feel that the authors' interpretation of their data is flawed in numerous areas. As space is restricted, I will only respond to the most serious problems. Although I agree that in respiring yeast the oscillation of metabolic events might be responsible for temporally partitioning cellular processes ${ }^{2,3}$, the authors seem to have compounded a series of fundamental errors in interpreting their data. The authors have used the reasoning that the inflection point of the transition between high and low residual dissolved oxygen states is when maximum respiratory activity occurs ${ }^{4}$. However, this is not true - when the measurements of off-gas concentrations of oxygen and carbon dioxide are taken, the exact oxygenconsumption rates can be calculated ${ }^{5-7}$ (FIG. 1). Clearly, maximum oxygen-consumption rates occur when the dissolved oxygen concentration is at a minimum. As respiratory activity is measured by oxygenconsumption rates, this means that the authors have incorrectly assigned the oxidative and reductive phases; this has serious implications regarding their discussion.

We conducted a similar study - in which we showed that the transcriptome oscillates with a shorter period (40 $\mathrm{min})$ and

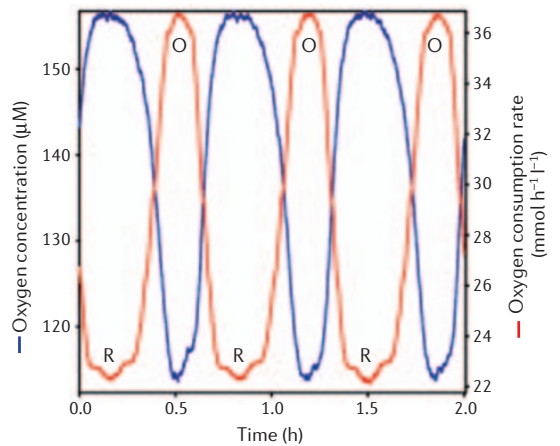

Figure 1 | The relationship between dissolved oxygen and oxygen-consumption rate during continuous culture of yeast. $O$ is the oxidative phase when the oxygen-consumption rate is at a maximum. $R$ is the reductive phase when the oxygen-consumption rate is at a minimum. Note that respiration always occurs. arrived at similar conclusions - that was not discussed in any detail in the authors' paper. This is surprising considering that these two papers have clear similarities ${ }^{8}$. Apart from the period, perhaps the principal difference of our findings was that the transcripts produced in the preceding phase were responsible for the resulting phenotype. When one factors in the authors' phase-calculation error, the same conclusion can be drawn from their data. A simple phase analysis of the top oscillating transcripts shows that both data sets are in-phase (FIG. 2), except for one minor cluster. Therefore, a naive interpretation is that the oscillation is a slowed down version of the 40-min cycle; but the situation is more complex.

A clue regarding the differences in period might be derived from a reduction in growth yield, which could result from differences in culture conditions. The short-period oscillation has a cell number that is roughly 5-6 fold higher than the long-period oscillation ${ }^{2}$. Although this could be caused by a number of factors, I am specifically concerned about potential reactor pressure caused by using an aeration rate of $1,000 \mathrm{~mL}$ per min. As the oscillation is thought to stem from individual cells rather than being caused by population effects, a reduction in cell density would increase the communication distance between the cells. As population synchrony is a function of communication distance ${ }^{9}, \mathrm{I}$ reason that this might be a determinant of periodicity. An increase in cell-cell communication distance or a disruption of synchronization mechanisms might also explain why mutations in central metabolic enzymes, such as glucose-6-phosphate dehydrogenase, result in the loss of respiratory oscillation in the population. However, this does not necessarily indicate that an individual cell has lost its oscillatory behaviour, but rather that cell-cell synchrony cannot be achieved ${ }^{9}$. So, although new and refreshing opinions are important for the progression of science, these opinions need to be based on solid published science and open discussion of relevant work in order to be validated.

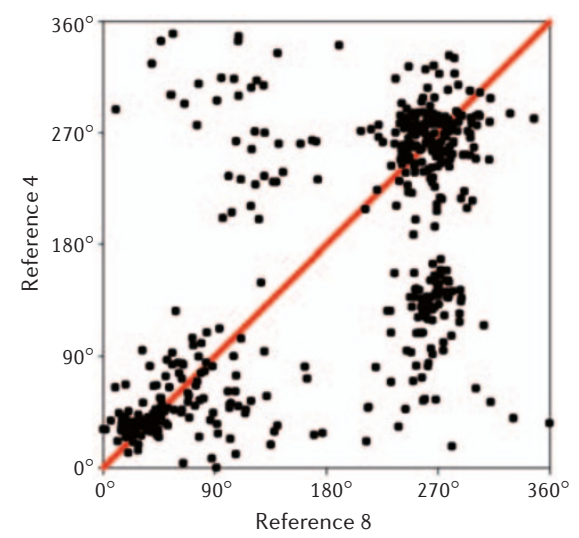

Figure 2 | A phase comparison by Fourier analysis of the data (from different studies) of the top oscillating genes (that is, those genes with the highest correlation to a sine wave). Data sets were used from REF. 4 and REF. 8. $0^{\circ}$ was derived from the minimum first derivative of the dissolved oxygen concentration. This corresponds to the beginning of the oxidative phase. The reductive phase begins at the maximum first derivative of the dissolved oxygen trace $\left(\sim 120^{\circ}\right)$. The red line represents a 1:1 correlation.

Systems Biology Institute, 9s3 Shinanomachi Research Park, Keio University School of Medicine, 35 Shinanomachi, Shinjuku-ku, Tokyo 160-8582, Japan. e-mail:dougie@symbio.jst.go.jp

doi: 10.1038/nrm1980-c2

Tu, B. P. \& McKnight, S. L. Metabolic cycles as an underlying basis of biological oscillations. Nature Rev. Mol. Cell Biol. 7, 696-701 (2006).

2. Lloyd, D. \& Murray, D. B. Ultradian metronome: timekeeper for orchestration of cellular coherence. Trends Biochem. Sci. 30, 373-377 (2005).

3. Lloyd, D. \& Murray, D. B. The temporal architecture of eukaryotic growth. FEBS Lett. 580, 2830-2835 (2006).

4. Tu, B. P., Kudlicki, A., Rowicka, M. \& McKnight, S. L. Logic of the yeast metabolic cycle: temporal compartmentalization of cellular processes. Science 310, 1152-1158 (2005).

5. Doran, P. A. Bioprocess Engineering (Academic Press Limited, London, 1995).

6. Keulers, M., Satroutdinov, A. D., Suzuki, T. \& Kuriyama, H. Synchronization affector of autonomous short-period-sustained oscillation of Saccharomyces cerevisiae. Yeast 12, 673-682 (1996).

7. Xu, Z. \& Tsurugi, K. A potential mechanism of energymetabolism oscillation in an aerobic chemostat culture of the yeast Saccharomyces cerevisiae. FEBS J. 273, 1696-1709 (2006).

8. Klevecz, R. R., Bolen, J., Forrest, G. \& Murray, D. B. A genomewide oscillation in transcription gates DNA replication and cell cycle. Proc. NatI Acad. Sci. USA 101, 1200-1205 (2004).

9. Winfree, A. T. The Geometry of Biological Time (Springer Verlag, Berlin, 2001). 